\title{
Comparative Analysis of the 2016 ACR-EULAR and the 2002 AECG Classification Criteria for Sjögren's Syndrome: Findings from the NIH cohort
}

\author{
Monisha Billings, DDS, MPH, PhD ${ }^{1}$, Mohammad Amin Hadavand, BS ${ }^{1}$, and llias Alevizos, \\ DMD, PhD 1 \\ ${ }^{1}$ National Institute of Dental and Craniofacial Research, National Institutes of Health, Bethesda, \\ Maryland, USA
}

\section{Abstract}

Introduction-The introduction of new classification criteria for Sjögren's Syndrome, known as the 2016 American College of Rheumatology/European League against Rheumatism Classification Criteria (ACR-EULAR), created a need for the evaluation of its performance in an external cohort. The purpose of this study was to compare the performance of the 2016 ACREULAR classification set with the widely used American-European Consensus Group Classification criteria (AECG) in the cohort at the National Institutes of Health, USA and to compare the performance of the sets in classifying both primary and secondary Sjögren's Syndrome (pSS and sSS).

Methods-The study cohort at the NIH (N=1303) was enrolled for clinical suspicion of SS. Participants were classified as SS, pSS, and sSS according to both classification sets. Performance of 2016 ACR-EULAR and AECG sets were compared holding each as gold standard to the other. Statistical analysis of test diagnostics and agreement between the two sets were undertaken.

Results-By the AECG set, 701 were classified as having SS (627 pSS, 74 sSS) and 714 were classified with SS (647 pSS, 67 sSS) by the 2016 ACR-EULAR set. Sensitivity and specificity of the two sets were comparable in classifying SS, pSS, and sSS. There was high agreement between the two sets for classifying SS $(K=0.79)$, pSS $(K=0.81)$, and sSS $(K=0.87)$. The specificity of the 2016 ACR-EULAR set was significantly higher for classifying sSS than pSS, while the sensitivity was similar for the two disease groups. However, this pattern was also exhibited by the AECG set.

Conclusion-There was high agreement between the two classification sets with comparable performance diagnostics. There was no evidence of superior performance value by the new 2016 ACR-EULAR set over the AECG set and the two sets were found to be equivalent. Findings from our cohort indicate that 2016 ACR-EULAR classification could be extended to classification of sSS.

Correspondence to: Dr. Ilias Alevizos, NIDCR, NIH, Building 10, Room 1N110, MSC-1190, Bethesda, MD 20892 USA, Tel: +1-301-594-1193, alevizosi@mail.nih.gov.

DR ILIAS ALEVIZOS (Orcid ID: 0000-0001-5331-1652)

Conflicts of Interest

None to declare 


\section{Introduction}

Sjögren's syndrome (SS) is a chronic, systemic autoimmune disorder that affects exocrine glands leading to dryness of mucosal surfaces, with oral and ocular dryness predominantly manifesting. In addition, the syndrome may present with numerous extraglandular manifestations. Such diverse manifestations make SS a complex pathology to characterize and gives rise to variability in disease classification, making comparability of research findings a challenge. Therefore, the need for a uniform, standardized set of criteria to classify individuals to have SS is imperative. There have been 13 classification criteria systems for SS from 1965 to 2016 (Bloch et al., 1965, Shearn, 1971, Daniels et al., 1975, Ohfuji, 1977, Manthorpe et al., 1981, Homma et al., 1986, Skopouli et al., 1986, Fox et al., 1986, Vitali et al., 1993, Vitali et al., 2002, Shiboski et al., 2012, Shiboski et al., 2017, Fujibayashi, 2000). The 2002 American-European Consensus Group Classification criteria (AECG) has been the most widely used classification set with over 1500 citations (Rasmussen et al., 2014). This set employed 6 domains of assessment, of which, 2 were subjective (ocular and oral dryness) and 4 were objective (ocular signs, oral signs, histopathology, and autoantibodies)(Vitali et al., 2002). However, in 2012 a new classification system known as the American College of Rheumatology (ACR) Classification Criteria for SS was proposed to improve specificity that would consequently limit the exposure of individuals (falsely classified as SS) to biologics of uncertain safety in clinical trials (Shiboski et al., 2012). This system eliminated symptoms of oral and ocular dryness and the objective sign of hyposalivation, and was based exclusively on objective signs of positive focus score, serology and ocular staining score. When the performance of this system was evaluated, it was found to be comparable with the AECG classification system in terms of sensitivity, specificity, and other test diagnostics and did not provide increased value over the existing AECG set (Rasmussen et al., 2014).

The 2012 ACR classification was replaced by another system proposed by the International Sjögren's Syndrome Criteria Working Group, known as the 2016 American College of Rheumatology/European League against Rheumatism Classification Criteria (ACREULAR) (Shiboski et al., 2017). This set incorporated criteria from both the AECG and ACR sets and gave weighted points for the four objective signs of focus score $\geq 1$ foci $/ 4 \mathrm{~mm}^{2}$ ( 3 points), anti-SSA/Ro-positive ( 3 points), ocular staining score $25 /$ Van Bijsterveld score ㄴ $(1$ point), and Schimer's test $5 \mathrm{~mm} / 5 \mathrm{~min}$ (1 point) and unstimulated whole saliva flow rate $₫ 0.1 \mathrm{ml} / \mathrm{min}$ (1 point). A total score of $\geq 4$ points classified someone as having primary SS (pSS) (Shiboski et al., 2017).

A recent study conducted with a Japanese cohort reported that the sensitivity of 2016 ACREULAR set was higher but the specificity was lower than that of the AECG, Japanese (JPN), and ACR sets. In addition, there was low agreement between the 2016 ACR-EULAR and the 3 other sets (Tsuboi et al., 2017). These findings contradicted previously reported performance estimates of the 2016 ACR-EULAR set (Shiboski et al., 2017). It has been suggested that the 2016 ACR-EULAR set developed for classification of pSS is also applicable to classify secondary SS (sSS). However, evidence confirming such an assumption is lacking and further research is needed, which has been acknowledged by the developers of the classification set (Shiboski et al., 2017). The study by Tsuboi et al (2017) 
with the Japanese cohort also restricted their analysis to pSS. Consequently, the performance of the 2016 ACR-EULAR in classifying sSS remained unknown. Therefore, given the contradictory performance estimates and existing lack of evidence of application of the 2016 ACR-EULAR set to sSS, the purpose of this study was not only to compare the performance of the 2016 ACR-EULAR classification criteria with the widely used AECG classification criteria in the cohort at the National Institutes of Health, USA, but also to compare the performance of the 2016 ACR-EULAR set with the AECG set in classifying both pSS and sSS.

\section{Methods}

\section{Study Population}

The NIDCR Sjögren's Syndrome and Salivary Gland Dysfunction Unit (SSGDU) has been evaluating and enrolling participants with dry mouth symptoms and/or clinical suspicion of SS since 1984, making the NIDCR/NIH SS cohort one of the largest in the US. To investigate the proposed objectives, data from the source cohort comprising participants enrolled before August 27, 2015 were analyzed. Details of the source cohort have been previously published (Billings et al., 2016).

\section{Classification}

In this study, participants were classified according to both classification sets, the AECG and 2016 ACR-EULAR sets. They were classified as SS (pSS \& sSS) and non-SS by applying the AECG Classification Criteria (inclusion and exclusion criteria). They were also classified by applying the 2016 ACR-EULAR Classification Criteria (screening, inclusion and exclusion criteria) for pSS and those with a potentially associated disease (such as a well-defined connective tissue disease), but otherwise met the 2016 ACR-EULAR criteria, were classified as sSS by this set. The sum of the number of pSS and sSS cases yielded the number of SS cases. Details of the clinical procedures have been previously published(Billings et al., 2016). All study participants provided Informed Consent prior to the initiation of any study procedures and clinical protocols were approved by an NIH Institutional Review Board, conforming to the standards indicated by the Declaration of Helsinki.

\section{Statistical Analysis}

Performance of the 2016 ACR-EULAR set was evaluated holding the AECG as gold standard. Conversely, performance of the AECG set was evaluated against the 2016 ACREULAR as gold standard in test diagnostics. Sensitivities and specificities of the classification sets were compared and tested by McNemar's test, Kappa statistic was computed, and receiver operating curves (ROC) were generated with area under the curve (AUC). Statistical significance was assessed on a set two-tailed p-value of 0.05. All statistical analyses were performed using STATA 14.0 (StataCorp. 2015. Stata Statistical Software: Release 14. College Station, TX: StataCorp LP). 


\section{Results}

The NIH cohort was comprised of participants with clinical suspicion of SS. When the AECG classification criteria were applied to this cohort ( $\mathrm{N}=1303), 701$ were classified as having SS and 602 did not meet criteria for SS. Of those with SS, 627 were classified as pSS and 74 as sSS (Table 1). When participants were classified according to the 2016 ACREULAR classification criteria ( $\mathrm{N}=1303)$, there were 714 SS cases and 589 did not meet criteria. Of those with SS, 647 were pSS and 67 were sSS. Of the 698 subjects who were classified as pSS by either set, 576 (83\%) met both classification sets. Similarly, of the 79 subjects who were classified as sSS by either set, $62(78 \%)$ met both classification sets.

The agreement between the two classification sets that is higher than what is expected by chance alone is estimated by the Kappa statistic. The agreement between the two sets in classifying SS was substantial $(K=0.79)$ which further improved for classifying pSS $(K=$ $0.81)$ and $\mathrm{sSS}(\mathrm{K}=0.87)$.

Performance of the two sets was evaluated in comparison with the other; with both held as gold standard in turns. The sensitivities, specificities, positive and negative predictive values of the classification sets are summarized in Table 2. The test diagnostics for classification of SS by the two classification sets were found to be comparable. The sensitivity of the 2016 ACR-EULAR was 91.0\% (95\%CI: 88.6\%-93.0\%) and that of the AECG was 89.4\% (95\%CI: 86.9\%-91.5\%). There was no statistically significant difference in sensitivities between the two sets ( $\mathrm{p}$-value $=0.31$ ). The 2016 ACR-EULAR had a slightly lower specificity of $87.4 \%$ (95\% CI: 84.5\%-89.9\%) but it was comparable with that of AECG $(89.3 \%, 95 \%$ CI: $86.5 \%-91.7 \%$, p-value $=0.31)$.

The 2016 ACR-EULAR classification set had a similar performance for the classification of pSS. The sensitivity of the set was 91.9\% (95\%CI: 89.4\%-93.9\%), which was comparable with the sensitivity of AECG $(89.0 \%, 95 \%$ CI: $86.4 \%-91.3 \%$, p-value $=0.09)$. Specificities of the two sets were also comparable with the 2016 ACR-EULAR having a specificity of 89.5\% (95\%CI: $86.9 \%-91.7 \%$ ) and the AECG with a specificity of $92.20 \%$ (95\%CI: $89.9 \%-94.2 \%$, p-value $=0.09)$.

The performance of the 2016 ACR-EULAR for classification of sSS was also evaluated. The classification set had a slightly lower sensitivity in detecting sSS than pSS with estimates of 83.80\% (95\%CI: 73.4\%-91.3\%) and 91.90\% (95\% CI: 89.4\%-93.9\%) respectively, though not statistically significantly different. However, the set had a significantly higher specificity for sSS (99.60\%, 95\%CI: 99.1\%-99.9\%) than pSS (89.50\%, 95\%CI: 86.9\%-91.7\%). Test diagnostics of the 2016 ACR-EULAR and AECG for sSS when compared, were found to be comparable (p-value 0.14). The sensitivity of the AECG for sSS was 92.50 (95\%CI: 83.4\%$97.5 \%)$ and the specificity was $99.0 \%$ (95\%CI: $98.3 \%-99.5 \%)$.

The area under the curve (AUC) for the new classification was estimated. The AUC for 2016 ACR-EULAR classification for SS was 0.89 (95\%CI: 0.87-0.91), for pSS was 0.91 (95\% CI:0.89-0.92), and for sSS was $0.92(0.87-0.96)$ and the three estimates were similar (Figure 1). 
We then increased the cut off of the 2016 ACR-EULAR classification criteria from a total score of $\geq 4$ to $\geq 5$ and evaluated its performance in classification of SS, particularly pSS. It was found that the sensitivity of the classification set for SS significantly dropped from $91 \%$ (95\%CI: $88.6 \%-93.0 \%$ ) to $67.9 \%$ (95\%CI: 64.3\%-71.3\%) (p<0.001) but the specificity significantly increased from $87.4 \%$ (95\%CI: $84.5 \%-89.9 \%$ ) to $96.5 \%$ (95\%CI: $94.7 \%-$ 97.8\%) ( $\mathrm{p}<0.001)$. Consequently, the classification's AUC for SS significantly decreased from 0.89 (95\% CI: 0.87-0.91) to 0.82 (95\%CI: 0.80-0.84). For pSS, when the cut off was increased to 25 , the sensitivity significantly dropped from $91.9 \%$ (95\% CI: $89.4 \%-93.9 \%$ ) to 68.7\% (95\%CI: 64.9\%-72.4\%) ( $<<0.001)$ and the specificity significantly increased from 89.5\% (95\% CI: $86.9 \%-91.7 \%$ ) to $97.2 \%$ (95\%CI: 95.6\%-98.3\%) (p<0.001). Here again, the AUC significantly decreased from 0.91 (95\% CI:0.89-0.92) to 0.83 (95\% CI: 0.81-0.85).

\section{Discussion}

Classification criteria are used to define a disease class for clinical trials and epidemiologic studies, and thereby to facilitate standardization and comparability of findings across studies. The closer the percentage of sensitivity (true positive rate) and specificity (true negative rate) of the criteria are to $100 \%$, the higher the diagnostic value of the criteria. However, due to the lack of uniformity in signs and symptoms in a disorder marked with heterogeneity of manifestations, development of classification criteria is challenging. This is particularly the case with Sjögren's syndrome, a complex autoimmune, systemic disorder.

The AECG introduced in 2002 originated from a revised version of the European criteria by the American-European Consensus Group (Vitali et al., 2002). It was found that the sensitivity of the AECG set was $97.4 \%$ and specificity was $89.4 \%$ for pSS when any four of the six domains were applied. Further, when obligatory criteria were applied, with exclusion of cases in which both serology and histopathology were negative, the sensitivity dropped to $89.5 \%$ and specificity increased to $95.2 \%$. As a higher specificity was more desirable for the purpose of classification, the obligatory criteria were introduced (Vitali et al., 2002).

In 2012 a new classification set (ACR) was proposed. However, when the performance of this new set was evaluated in external cohorts, it was found to be comparable with the existing AECG set and no increased value was exhibited by the new set (Rasmussen et al., 2014). The study conducted on a cohort of 646 individuals in Oklahoma and Minnesota, found the sensitivity of the ACR set in classifying SS was 87\% (95\%CI: 83\%-91\%) and the specificity was $93 \%$ (95\%CI: $90 \%-96 \%$ ), while the sensitivity of the AECG set was $91 \%$ (95\%CI: 87\%-94\%) and specificity was 91\% (95\%CI: 87\%-93\%). The study found no statistically significant difference in these diagnostic values $(\mathrm{p}=0.19)$ and there was strong agreement between the two sets ( $\mathrm{K}=0.81$ ) (Rasmussen et al., 2014).

The 2012 ACR set was replaced by the revised 2016 ACR-EULAR set. This revised set was recently evaluated on a cohort of 499 (383 sub-cohort) Japanese patients for classification of pSS (Tsuboi et al., 2017). The study found that the sensitivity (94.1\%, 95\%CI: 90.8\%-96.4) of the 2016 ACR-EULAR set was significantly higher than that of the AECG $(85.7 \%$, 95\%CI: $82.1 \%-88.7 \%)$, but the specificity $(76.7,95 \%$ CI: $73.0 \%-79.3 \%)$ was significantly lower than that of the AECG $(86.1 \%, 95 \%$ CI: $82.0 \%-89.4 \%)$, (p<0.001) (Tsuboi et al., 
2017). The low agreement between the two sets was contrary to previously published estimates (Shiboski et al., 2017). These controversial findings were attributed to the difference in prevalence estimates in Japan as opposed to geographic settings where the classification set was developed and to insufficient investigation of sicca symptoms in the cohort (Vitali \& Del Papa, 2017).

To evaluate the performance of the 2016 ACR-EULAR classification set in comparison to AECG, we undertook a comparative analysis in an external cohort at the NIH, USA with detailed evaluation of sicca symptoms and objective signs mandated by both sets of classification. In our study cohort $(\mathrm{N}=1303)$, the performance of the two sets was comparable in classifying SS, pSS and sSS with very high agreement. Although we could see the pattern indicated by the Japanese study of higher sensitivity and lower specificity of the 2016 ACR-EULAR set compared to that of the AECG, it was not of statistical significance. The reason for a pattern of increased sensitivity (though not significant in our cohort) could be potentially explained by the influence of weights attributed by the 2016 ACR-EULAR set, and because the 2016 ACR-EULAR set only requires two objective signs, with either histopathology or serology being positive, while the AECG requires four positive criteria. For example, an individual with only a focus score $>=1$ foci $/ 4 \mathrm{~mm}^{2}$ and whole unstimulated salivary flow $\unlhd .5 \mathrm{ml} / 15 \mathrm{~min}$ will be assigned a total score of 4 , which classifies the person as SS with the 2016 ACR-EULAR set. Yet this is insufficient to classify as SS by AECG, which would require an additional two signs or symptoms.

This study undertook evaluation of the 2016 ACR-EULAR set in classifying not only pSS but also sSS, which has not been evaluated thus far. The findings indicate that the sensitivity of 2016 ACR-EULAR for pSS and sSS were comparable. However, the specificity of 2016 ACR-EULAR for sSS was statistically significantly higher than the set's specificity for pSS. Nevertheless, such a pattern was also exhibited by the AECG set. The sensitivity and specificity for sSS between the two sets were comparable, indicating that the 2016 ACREULAR set could be applied to classification of sSS.

Finally, as the study with the Japanese cohort found that the 2016 ACR-EULAR set had a low specificity compared to other classifications, we explored the impact of increasing the classifying cutoff of the 2016 ACR-EULAR from $\geq 4$ to $\geq 5$. We found that when the cut off increased to $>=5$, the specificity of the 2016 ACR-EULAR increased but its sensitivity decreased and the overall test performance was lower compared to its performance with a cut off of $>=4$. Therefore, the cut off of $>=4$ recommended by the developers of the classification set showed to be more efficient than a cut off of $>=5$.

In conclusion, in the NIH cohort, the evaluation of the 2016 ACR-EULAR classification set had a comparable performance to the AECG set. Test diagnostics such as sensitivity and specificity of the 2016 ACR-EULAR set were not significantly different from that of the AECG set. Further research to attain the objective of developing improved classification criteria with increased specificity is needed. In that respect, the assessment of the effect of increasing the existing focus score cut off of $1 \mathrm{foci} / 4 \mathrm{~mm}^{2}$, continuing research in understanding the underlying etiopathogenesis and possibly incorporating genetic and 
genomic alterations might prove beneficial in constructing improved criteria for this complex disorder.

\section{Acknowledgments}

This research was supported by the Intramural Research Program of the NIH, NIDCR. We would also like to acknowledge the members of the Sjögren's Syndrome Clinic at NIDCR and our Sjögren's syndrome subjects for their participation in our studies.

Funding Source: This research was supported by the Intramural Research Program of the National Institutes of Health (NIH), National Institute of Dental and Craniofacial Research (NIDCR).

\section{References}

Billings M, Dye BA, Iafolla T, Baer AN, Grisius M, Alevizos I. Significance and Implications of Patient-reported Xerostomia in Sjogren's Syndrome: Findings From the National Institutes of Health Cohort. EBioMedicine. 2016; 12:270-279. [PubMed: 27639822]

Bloch KJ, Buchanan WW, Wohl MJ, Bunim JJ. Sjoegren's Syndrome. A Clinical, Pathological, and Serological Study of Sixty-Two Cases. Medicine. 1965; 44:187-231. [PubMed: 14315274]

Daniels TE, Silverman S Jr, Michalski JP, Greenspan JS, Sylvester RA, Talal N. The oral component of Sjogren's syndrome. Oral Surg Oral Med Oral Pathol. 1975; 39:875-85. [PubMed: 1055974]

Fox RI, Robinson CA, Curd JG, Kozin F, Howell FV. Sjogren's syndrome. Proposed criteria for classification. Arthritis and rheumatism. 1986; 29:577-85. [PubMed: 3718551]

Fujibayashi T. Revised diagnostic criteria for Sjögren's syndrome. Rheumatology (Oxford). 2000; 24:421-428.

Homma M, Tojo T, Akizuki M, Yamagata H. Criteria for Sjogren's syndrome in Japan. Scand J Rheumatol Suppl. 1986; 61:26-7. [PubMed: 3473640]

Manthorpe R, Frost-Larsen K, Isager H, Prause JU. Sjogren's syndrome. A review with emphasis on immunological features. Allergy. 1981; 36:139-53. [PubMed: 7015910]

Ohfuji T. Summary of the studies on Sjogren's disease in 1977. Annual report of the ministry of Health and Welfare: Sjögren's disease Research Committee. 1977:3-6.

Rasmussen A, Ice JA, Li H, Grundahl K, Kelly JA, Radfar L, Stone DU, Hefner KS, Anaya JM, Rohrer M, Gopalakrishnan R, Houston GD, Lewis DM, Chodosh J, Harley JB, Hughes P, MaierMoore JS, Montgomery CG, Rhodus NL, Farris AD, Segal BM, Jonsson R, Lessard CJ, Scofield RH, Sivils KL. Comparison of the American-European Consensus Group Sjogren's syndrome classification criteria to newly proposed American College of Rheumatology criteria in a large, carefully characterised sicca cohort. Ann Rheum Dis. 2014; 73:31-8. [PubMed: 23968620]

Shearn MA. Sjogren's syndrome. Major Probl Intern Med. 1971; 2:1-262. [PubMed: 4949970]

Shiboski CH, Shiboski SC, Seror R, Criswell LA, Labetoulle M, Lietman TM, Rasmussen A, Scofield H, Vitali C, Bowman SJ, Mariette X, International Sjogren's Syndrome Criteria Working G. 2016 American College of Rheumatology/European League Against Rheumatism classification criteria for primary Sjogren's syndrome: A consensus and data-driven methodology involving three international patient cohorts. Ann Rheum Dis. 2017; 76:9-16. [PubMed: 27789466]

Shiboski SC, Shiboski CH, Criswell L, Baer A, Challacombe S, Lanfranchi H, Schiodt M, Umehara H, Vivino F, Zhao Y, Dong Y, Greenspan D, Heidenreich AM, Helin P, Kirkham B, Kitagawa K, Larkin G, Li M, Lietman T, Lindegaard J, McNamara N, Sack K, Shirlaw P, Sugai S, Vollenweider C, Whitcher J, Wu A, Zhang S, Zhang W, Greenspan J, Daniels T, Sjogren's International Collaborative Clinical Alliance Research G. American College of Rheumatology classification criteria for Sjogren's syndrome: a data-driven, expert consensus approach in the Sjogren's International Collaborative Clinical Alliance cohort. Arthritis Care Res (Hoboken). 2012; 64:47587. [PubMed: 22563590]

Skopouli FN, Drosos AA, Papaioannou T, Moutsopoulos HM. Preliminary diagnostic criteria for Sjogren's syndrome. Scand J Rheumatol Suppl. 1986; 61:22-5. [PubMed: 3473635] 
Tsuboi H, Hagiwara S, Asashima H, Takahashi H, Hirota T, Noma H, Umehara H, Kawakami A, Nakamura H, Sano H, Tsubota K, Ogawa Y, Takamura E, Saito I, Inoue H, Nakamura S, Moriyama M, Takeuchi T, Tanaka Y, Hirata S, Mimori T, Matsumoto I, Sumida T. Comparison of performance of the 2016 ACR-EULAR classification criteria for primary Sjogren's syndrome with other sets of criteria in Japanese patients. Ann Rheum Dis. 2017

Vitali C, Bombardieri S, Jonsson R, Moutsopoulos HM, Alexander EL, Carsons SE, Daniels TE, Fox PC, Fox RI, Kassan SS, Pillemer SR, Talal N, Weisman MH, European Study Group on Classification Criteria for Sjogren's S. Classification criteria for Sjogren's syndrome: a revised version of the European criteria proposed by the American-European Consensus Group. Ann Rheum Dis. 2002; 61:554-8. [PubMed: 12006334]

Vitali C, Bombardieri S, Moutsopoulos HM, Balestrieri G, Bencivelli W, Bernstein RM, Bjerrum KB, Braga S, Coll J, de Vita S, et al. Preliminary criteria for the classification of Sjogren's syndrome. Results of a prospective concerted action supported by the European Community. Arthritis and rheumatism. 1993; 36:340-7. [PubMed: 8452579]

Vitali C, Del Papa N. Classification and diagnostic criteria in Sjogren's syndrome: a long-standing and still open controversy. Ann Rheum Dis. 2017 


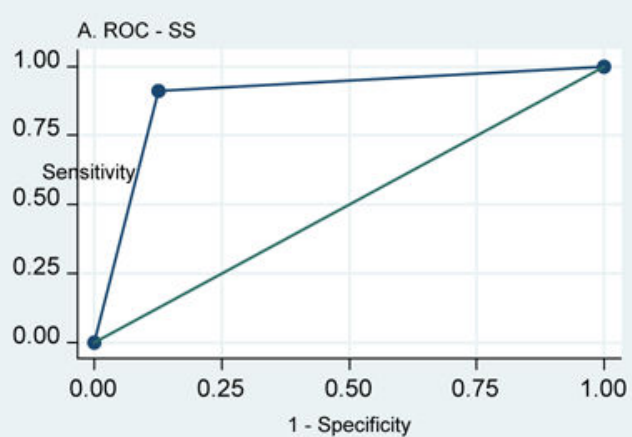

Area under $\mathrm{ROC}$ curve $=0.8919$

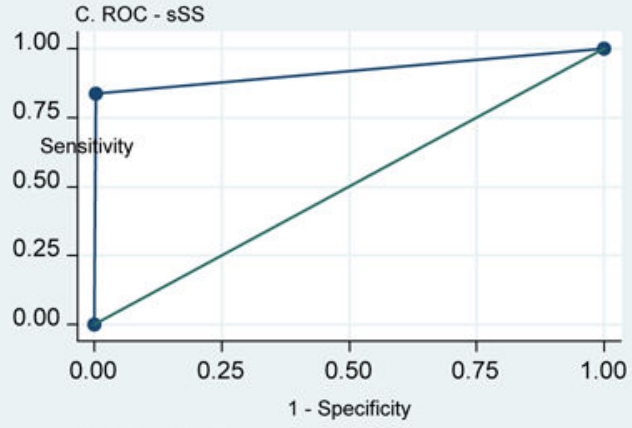

Area under $\mathrm{ROC}$ curve $=0.9169$

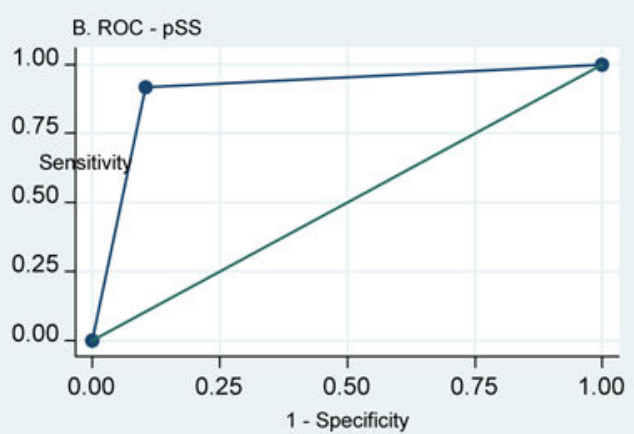

Area under $\mathrm{ROC}$ curve $=0.9068$

Figure 1.

Receiver Operating Curves (ROC) of 2016 ACR-EULAR Classification of SS, pSS and sSS. 

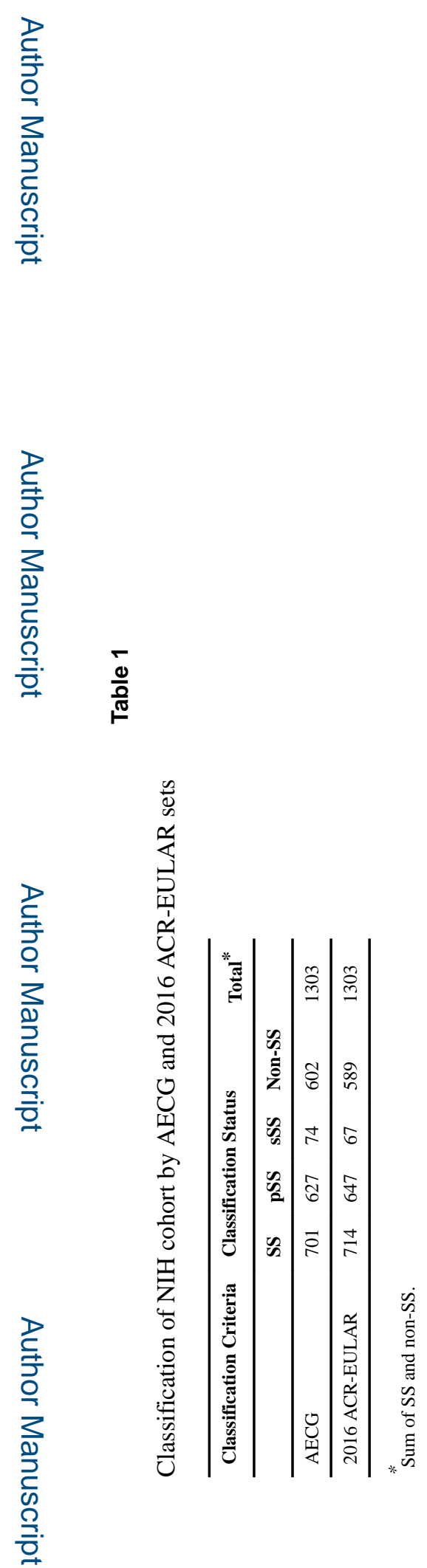

Oral Dis. Author manuscript; available in PMC 2019 March 01. 


\section{롤 \\ 골}

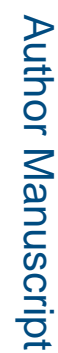

?

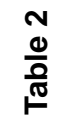

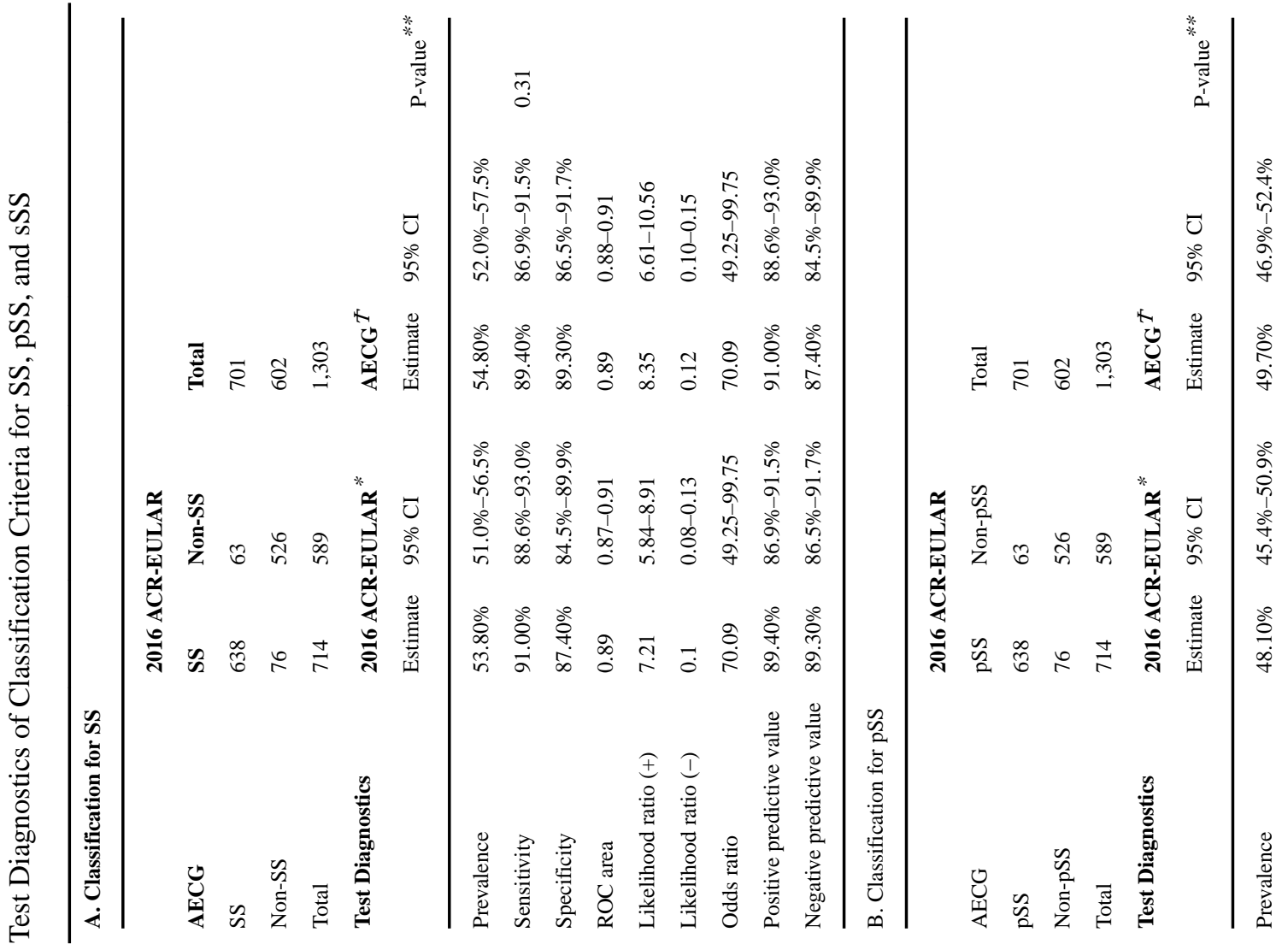
Oral Dis. Author manuscript; available in PMC 2019 March 01. 
Billings et al.

Page 12

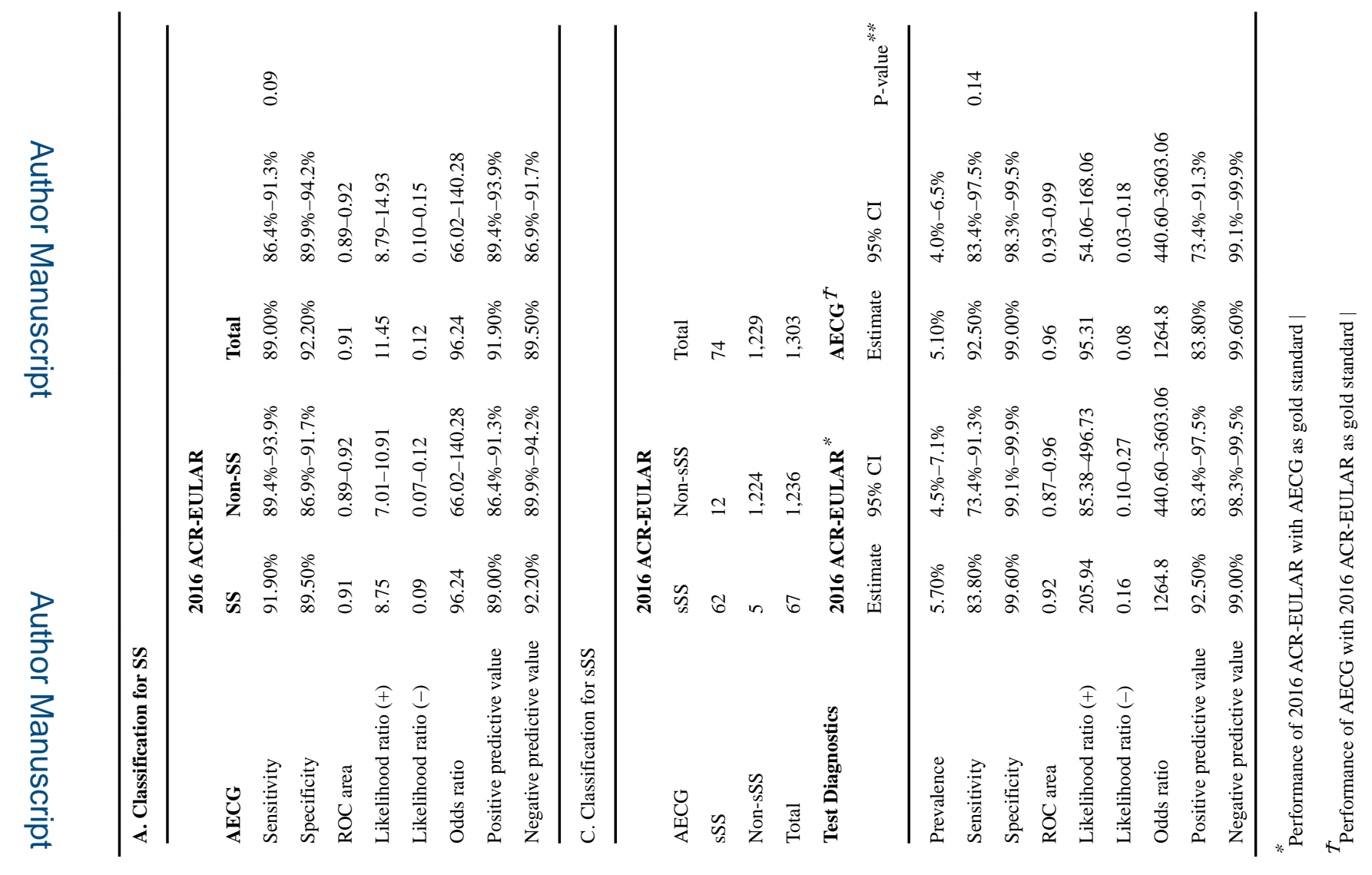

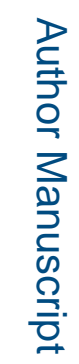

로을

Oral Dis. Author manuscript; available in PMC 2019 March 01. 
